\title{
Putting the magic in magic bullets: top three global priorities for sexually transmitted infection control
}

\author{
Nicola Low, ${ }^{1}$ Sarah J Hawkes ${ }^{2}$
}

\begin{abstract}
${ }^{1}$ Division of Clinical
Epidemiology and Biostatistics, Institute of Social and Preventive Medicine, University of Bern, Bern, Switzerland ${ }^{2}$ Institute for Global Health, University College London, London, UK
\end{abstract}

\section{Correspondence to}

Professor Nicola Low, Division of Clinical Epidemiology and

Biostatistics, Institute of Social and Preventive Medicine, University of Bern, Finkenhubelweg 11, Bern CH-3012, Switzerland; low@ispm.unibe.ch

Accepted 15 September 2011

This paper is freely available online under the BMJ Journals unlocked scheme, see http://sti. bmi.com/site/about/unlocked. xhtml

\author{
"Man is born, not to solve the problems of the \\ universe, but to find out where the problem applies, \\ and then to restrain himself within the limits of the \\ comprehensible." Goethe, 1825
}

\section{INTRODUCTION}

Setting practical priorities for sexually transmitted infection (STI) control is a balance between idealism and pragmatism. Infections transmitted through unsafe sex (chlamydia, gonorrhoea, syphilis, HIV, hepatitis B and human papillomavirus (HPV) infections) rank in the top five causes of the global burden of disease. ${ }^{1}$ Their distribution in populations is driven by a complex mixture of individual behaviours, social and community norms and societal and historical context. Ideally, we would be able to reduce exposure to unsafe sex to its theoretical minimum level of zero and thus eliminate a significant proportion of the current global burden of disease, particularly in resourcepoor settings. ${ }^{2}$ Ideally, we would have 'magic bullets' for diagnosing and preventing STI in addition to specific antimicrobial agents for specific infections. ${ }^{3}$ Arguably, we have 'bullets' that work at the individual level; highly accurate diagnostic tests and highly efficacious vaccines, antimicrobial agents and preventive interventions. ${ }^{4}$ Introducing them into populations to achieve similarly high levels of effectiveness has been more challenging. ${ }^{4}$ In practice, the 'magic' in the magic bullet can be seen as overcoming the barriers to sustainable implementation in partnerships, larger sexual networks and populations (figure 1). ${ }^{4}$ We have chosen three (pragmatic) priorities for interventions that we believe could be implemented and scaled up to control STI other than HIV/AIDS. We present these starting with the partnership and moving up to the population level.

\section{STRENGTHEN PARTNER NOTIFICATION}

Strengthening the implementation and effectiveness of partner notification has the potential to contribute to the control of several STI. Partner notification is done for different reasons and its use and evaluation need to take these into consideration. First, the prevalence of infection in sexual partners who attend for testing is high, so partner notification is efficient for case finding. Second, partner notification can prevent re-infection from an existing partner with a curable STI, in which case the couple should not have unprotected sex until the infection is eradicated. Ineffective partner notification is one reason for the high levels of repeated chlamydia observed after treatment. Third, partner notification could interrupt the onward transmission in a sexual network; a notified partner would then bring another infected partner to treatment and avoid having sex until their own infection is cleared.

The traditional debate focuses on whether sexual partners should be informed by the index patient (patient referral), a healthcare professional (provider referral) or a combination in which the provider takes responsibility for informing partners if the index case is unable to (contract referral). In practice, patient referral is preferred by both patients and providers, and is often the only affordable method available, particularly for the most common STI managed outside specialist health services. The content of patient referral is rarely defined and, at its simplest, consists of advice for the index patient at the end of a consultation, such as 'You should tell your partner(s) that they need treatment too'. Sexual partner histories are often not elicited, supporting materials are not given and there might be no follow-up. ${ }^{5}$

Randomised controlled trials show that index cases with gonorrhoea or chlamydia who are given additional infection-specific written information for their partner(s), receive additional counselling or both are up to $50 \%$ less likely to have repeated infections at follow-up than those receiving simple patient referral. ${ }^{6} 7$ The effectiveness of these enhancements is at least as good as that observed for expedited partner therapy, ${ }^{6}$ which might not be acceptable because of the risk that sexual partners would miss out on access to preventive services. ${ }^{7}$ It is possible that the additional benefits of both enhanced patient referral and expedited partner therapy in trials are related more to the more intensive communication and interaction with the patient than specific effects of the methods. Further trials would therefore help to identify the essential components of enhanced patient referral, including the use of newer technologies such as text messaging and the web, and determine their effectiveness in resource-limited settings. Ideally, we would like to have empirical data to show the extent to which partner notification reduces the prevalence or incidence of STI, but modelling studies might be the only way to obtain this information. ${ }^{8}$

A priority for improving partner notification outcomes is the allocation of resources to implement it as an integral part of the case management of STI. Clinical guidelines need to be actively disseminated to all healthcare professionals who diagnose and treat STI and include recommendations about appropriate contact periods, advice, follow-up and protection of patient confidentiality. At the same time, further work is needed to reduce 


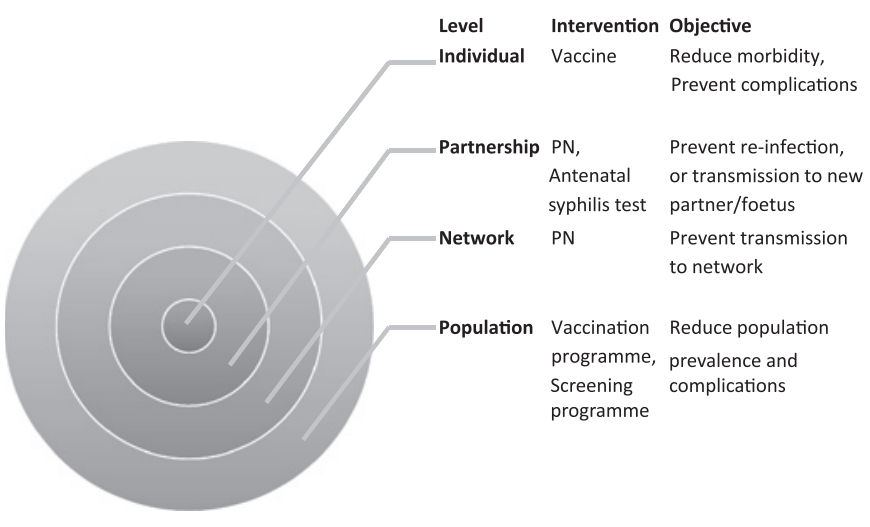

Figure 1 Levels of action for sexually transmitted infection interventions. Adapted from Low et al. ${ }^{4}$ PN, partner notification.

the barriers for providers in initiating partner notification (including embarrassment and lack of training and time) and for patients in informing and referring partners (including embarrassment, fear of relationship breakdown or partner violence, and concerns about confidentiality).

\section{IMPLEMENT ANTENATAL SYPHILIS SCREENING PROGRAMMES}

Congenital syphilis is at least as important a cause of fetal and perinatal mortality as HIV infection. ${ }^{9}$ Syphilis, however, rarely achieves a position of salience within antenatal and child health programmes, even though there are an estimated two million women every year with active syphilis in pregnancy. ${ }^{9}$ Untreated or inadequately treated syphilis in pregnancy is estimated to result in adverse pregnancy outcomes in up to $69 \%$ of infected women, including late abortion (after 16 weeks) or stillbirth in $25 \%$, prematurity or low birth weight in $13 \%$, neonatal death in $11 \%$ and clinical congenital syphilis in $20 \%$. ${ }^{9}$ Most countries have antenatal syphilis screening policies in place but implementation is poor. A major challenge is that few women in higher prevalence areas (particularly in Africa) attend antenatal clinics before 20 weeks' gestation. ${ }^{10}$ Currently, it is estimated that fewer than one in eight women receives syphilis testing at any point in their pregnancy. ${ }^{11}$

We have the evidence to show the potential benefi ts of antenatal syphilis screening interventions. Antenatal syphilis screening interventions are a package of 'bullets' such as pointof-care tests and single-dose antibiotic treatment that need to be combined with: early antenatal clinic attendance; decentralisation and same-day treatment; partner notification; third trimester retesting; and strengthening of health services to provide supplies, training and monitoring. A meta-analysis of randomised and non-randomised trials comparing a range of interventions with usual care found reductions in perinatal death and stillbirth due to syphilis of approximately $50 \% .{ }^{11}$ The incidence of congenital syphilis was also reduced but with heterogeneous results. Women who have syphilis diagnosed in pregnancy and treated with a single dose of long-acting penicillin have the same rate of adverse pregnancy outcomes as seronegative women. ${ }^{10}$ The exact combination of activities needed to optimise effectiveness is not known.

There is already a WHO global initiative to eliminate congenital syphilis, which is based on the guiding principles of a country-driven, integrated, rights-based and collaborative strategy. The four pillar strategy involves: (1) ensuring advocacy and sustained political commitment for a successful health initiative; (2) increasing access to, and quality of, maternal and newborn health services; (3) screening and treating pregnant women and partners; and (4) establishing surveillance, monitoring and evaluation systems. ${ }^{9}$ Political support for the elimination of congenital syphilis is growing in some regions, for example, the Pan-American region, which has an integrated strategy for the elimination of congenital HIV infection and syphilis. A cornerstone of the strategy is to strengthen, integrate and decentralise services so that the successes of implementing the prevention of mother-to-child transmission of HIV can be broadened to include simultaneous access to diagnosis and treatment of syphilis in pregnancy. ${ }^{12}$ Nonetheless, there are still challenges to ensuring effective implementation, including universal access to early antenatal care, health systems strengthening to ensure adequate resources (financial, human, logistical) for screening, and raising awareness of congenital syphilis among health workers and pregnant women and their families.

\section{DELIVER VACCINES AGAINST STI}

Vaccines can protect individuals against infection or disease. To achieve the coverage levels needed to realise population-level benefits, however, vaccination needs to be delivered as an organised programme. We now have two highly efficacious vaccines that prevent the acquisition of hepatitis $B$ and HPV and, ultimately, liver cancer and high-grade precancerous lesions of the cervix. Hepatitis B was estimated to have caused 2 million disability-adjusted life-years and HPV-associated cervical cancer another 3.7 million disability-adjusted life-years lost in 2004. ${ }^{1}$ The first hepatitis B vaccine was tested on men who have sex with men in the 1970s but, paradoxically, was not subsequently strongly promoted for the prevention of STI. ${ }^{13} \mathrm{HPV}$ vaccines protecting against the two most common oncogenic serotypes (16 and 18) were licensed in 2007 and are projected to prevent up to $70 \%$ of cervical cancer cases, $80 \%$ of which occur in developing countries. ${ }^{14}$ HPV vaccination has been taken up rapidly, but in developed countries that already have strong cervical cancer screening programmes and a low burden of disease.

Universal hepatitis B vaccination in infancy is the only strategy that will eliminate sexual transmission. WHO has recommended this strategy since 1992 and it is now included in national immunisation programmes in 164 countries. The rate of chronic carriage has been substantially reduced in these countries and reduced sexual transmission should be seen soon. Some countries, including the UK, where the number of cases of acute hepatitis B (approximately 700 a year) has not been reduced, continue to recommend vaccination only for those at high risk of infection or of complications of disease. Ongoing transmission can continue with targeted vaccination because, even though $90 \%$ of men who have sex with men attending genitourinary medicine clinics have a first dose of hepatitis $B$ vaccine, only approximately $50 \%$ complete the course, and many others who become infected through heterosexual sex would not have been identified as being at high risk.

HPV vaccination is recommended by WHO as part of a coordinated strategy to prevent all HPV-related disease. ${ }^{14}$ There are major challenges for delivery to 11-14-year-old girls in settings in which school attendance is low, to overcome concerns about the use of a vaccine in children to prevent a STI, and to provide the financing for a new and very expensive vaccine. Screening for cervical cancer at older ages will also need to continue to detect lesions caused by HPV serotypes not included in the vaccines. The benefits are likely to be highest in developing countries with a high disease burden where cervical cancer screening is poor, 
such as India, which accounts for $25 \%$ of all cervical cancer cases worldwide. $^{15}$

\section{CONCLUSION}

The interventions prioritised here are of confirmed efficacy ${ }^{4} 13$ and all, apart from HPV vaccination, have been available for decades at least. The effective implementation of partner notification and antenatal syphilis screening are 'quick wins', while the population benefits of vaccination programmes for cancer prevention will take longer to realise. These are pragmatic and achievable interventions that could be implemented even in resource-poor settings as long as there is sufficient political commitment (including resource allocation) to tackling the burden of STI. In an ideal world, we should also be working to influence public policies to address the underlying drivers of STI epidemics, including poverty, gender inequality and lack of sexual rights. This will take time, resources and intersectoral collaboration, for example, by forming partnerships with the education, legal and employment sectors. With commitment, organisation and delivery at the appropriate levels, combinations of interventions will reduce the burden of STI substantially.

\section{Competing interests None.}

Provenance and peer review Commissioned; externally peer reviewed.

\section{REFERENCES}

1. World Health Organization. The Global Burden of Disease: 2004 Update. Geneva: World Health Organization, 2008. http://www.who.int/healthinfo/ global_burden_disease/2004_report_update/en/ (accessed 13 Sep 2011).
2. Ezzati M, Lopez $A D$, Rodgers $A$, et al. Selected major risk factors and global and regional burden of disease. Lancet 2002;360:1347-60.

3. Brandt AM. No Magic Bullet: A Social History of Venereal Disease in the United States Since 1880. New York: Oxford University Press, Inc, 1985.

4. Low N, Broutet N, Adu-Sarkodie Y, et al. Global control of sexually transmitted infections. Lancet 2006:368:2001-16.

5. St Lawrence JS, Montaño DE, Kasprzyk D, et al. STD screening, testing, case reporting, and clinical and partner notification practices: a national survey of US physicians. Am J Public Health 2002;92:1784-8.

6. Trelle S, Shang A, Nartey L, et al. Improved effectiveness of partner notification for patients with sexually transmitted infections: systematic review. BMJ 2007;334:354-7.

7. Wilson TE, Hogben M, Malka ES, et al. A randomized controlled trial for reducing risks for sexually transmitted infections through enhanced patient-based partner notification. Am J Public Health 2009;99(Suppl 1):S104-10.

8. Althaus CL, Heijne JC, Herzog SA, et al. Individual and population level effects of partner notification for Chlamydia trachomatis. Health services and policy oral session 3: Partner notification 05-S03.04. 19th Biennial Meeting of the International Society STD Research. 11-13 July 2011, Quebec City, Canada. Sex Transm Infect 2011;87 (Suppl 1):A95.

9. Kamb ML, Newman LM, Riley PL, et al. A road map for the global elimination of congenital syphilis. Obstet Gynecol Int 2010:312798. doi:10.1155/2010/312798.

10. Watson-Jones D, Gumodoka B, Weiss H, et al. Syphilis in pregnancy in Tanzania. II. The effectiveness of antenatal syphilis screening and single-dose benzathine penicillin treatment for the prevention of adverse pregnancy outcomes. J Infect Dis 2002; 186:948-57.

11. Hawkes SJ, Matin N, Broutet N, et al. Effectiveness of interventions to improve screening for antenatal syphilis: a systematic review and meta-analysis. Lancet Infect Dis 2011:11:684-91.

12. Panamerican Health Organization, World Health Organization. Strategy and Plan of Action for the Elimination of Mother-to-Child Transmission of HIV and Congenital Syphilis. Washington DC: PAH0, 2010:1-23. CD50/15 (Eng).

13. Manhart LE, Holmes KK. Randomized controlled trials of individual-level, populationlevel, and multilevel interventions for preventing sexually transmitted infections: what has worked? J Infect Dis 2005;191(Suppl 1):S7-24.

14. World Health Organization. Human papillomavirus vaccines-WHO position pape (In French). Wkly Epidemiol Rec 2009:84:118-31.

15. Arbyn M, Castellsagué $X$, de Sanjosé S, et al. Worldwide burden of cervical cancer in 2008. Ann Oncol. Published Online First: 6 April 2011. doi:10.1093/annonc/mdr015 